\title{
Intracerebral haemorrhage after thrombolysis for acute ischaemic stroke: an update
}

\author{
L Derex, N Nighoghossian
}

Service d'Urgences

Neurovasculaires, Hôpital

Neurologique, Lyon, France and CREATIS, UMR CNRS 5515,

Claude Bernard University,

INSERM, Lyon, France

Correspondence to:

Dr L Derex, Service d'Urgences

Neurovasculaires, Hôpital

Neurologique, 59 boulevard

Pinel, 69003 Lyon, France;

laurent.derex@chu-lyon.fr

Received 27 August 2007

Revised 10 December 2007

Accepted 10 December 2007

Published Online First

25 January 2008

\section{ABSTRACT}

Intracerebral haemorrhage (ICH) still represents the most feared complication of thrombolysis. Our aim was to review the literature regarding clinical, biological and imaging predictors of ICH following thrombolysis for acute ischaemic stroke. Relevant studies were identified through a search in Pubmed, using the following key words: "intracerebral", "haemorrhage", "stroke" and "thrombolytic". The query was limited to studies published in the English literature. The reference lists of all relevant articles were reviewed to identify additional studies. The main predictors of clinically significant ICH were age, clinical stroke severity, as assessed by the National Institute of Health Stroke Scale score on admission, high blood pressure, hyperglycaemia, early CT changes, large baseline diffusion lesion volume and leukoaraiosis on MRI. The contribution of biomarkers in the prediction of the ICH risk is currently under evaluation. Available data on patients with limited number of microbleeds on pretreatment gradient echo MRI sequences suggest safe use of thrombolysis. ICH after stroke thrombolysis is a complex and heterogeneous phenomenon, which involves numerous parameters whose knowledge remains partial. To minimise the risk of tissue plasminogen activator (tPA) related symptomatic ICH, careful attention must be given to the pretherapeutic glycaemia value, and a strict protocol for the control of elevated blood pressure is needed during the first $24 \mathrm{~h}$. Future research should focus on predictors of severe intracerebral haemorrhagic complications (parenchymal haematomas type 2 according to the European Cooperative Acute Stroke Study (ECASS) classification). The input of multimodal MRI and biological predictors of $\mathrm{ICH}$ deserves further investigation.

After publication of the results of the National Institute of Neurological Disorders and Stroke (NINDS) study, ${ }^{1}$ the application of intravenous thrombolysis for ischaemic stroke was launched and has now been in use for more than 10 years. This breakthrough ended decades of therapeutic nihilism but we have to acknowledge the fact that despite recommendations, only $1-8 \%$ of potentially eligible patients are currently being treated in the USA or in Europe. ${ }^{2}$ Several factors explain this situation: a therapeutic window currently limited to $3 \mathrm{~h}$, insufficient public knowledge of the stroke warning signs, the small number of centres able to administer thrombolysis on a $24 \mathrm{~h}$ basis and also an excessive fear of haemorrhagic complications from some physicians. ${ }^{3}$

Although it may finally be less frequent in practice than failure of treatment to reopen the occluded cerebral artery or early reocclusion, intracerebral haemorrhage (ICH) remains the most feared complication of stroke thrombolysis and an important obstacle to the generalisation of thrombolytic therapy. ICH occurs in the vast majority of cases in the core of the infarction, which suggests that ischaemia itself plays a role. ${ }^{4}$ Symptomatic ICHs usually occur within 24-36 h after thrombolysis, and ICHs that occur after $36 \mathrm{~h}$ are considered unrelated to tissue plasminogen activator (tPA). ${ }^{4}$ In the NINDS study, $6.4 \%$ (20/312) of patients showed a symptomatic ICH with deterioration of the clinical status in the tPA group compared with $0.6 \%$ in the placebo group. ${ }^{1}$ The mortality rate in cases of symptomatic ICH was $47 \%$ but the global mortality rate was lower in the tPA group than in the placebo group ( $17 \%$ vs $21 \%$ ) because of a reduction in death not related to haemorrhage. ${ }^{4}$ The other randomised studies of thrombolysis with intravenous tPA performed within a therapeutic window of 5-6 h provided a symptomatic ICH rate of $7.2 \%$ for the Alteplase Thrombolysis for Acute Noninterventional Therapy in Ischaemic Stroke (ATLANTIS) study ${ }^{5}$ and $8.8 \%$ for the European Cooperative Acute Stroke Study (ECASS) II. ${ }^{6}$ The symptomatic ICH rate was higher in the intra-arterial thrombolysis study, Prolyse in Acute Cerebral Thromboembolism II (PROACT II) $(10.9 \%)$ but the patients included in this study ${ }^{7}$ were more severely affected than those included in the NINDS, ATLANTIS and ECASS II studies (National Institute of Health Stroke Scale (NIHSS) median score of 17 vs 14,11 and 11, respectively). Moreover, direct comparison of the symptomatic ICH rates among different studies may be hampered by variability in definitions.

The influence of delay before treatment and the impact of arterial recanalisation on the haemorrhagic risk after thrombolysis remain controversial..$^{-13}$ The majority of open studies of thrombolysis with intravenous tPA performed within $3 \mathrm{~h}$ have confirmed the symptomatic ICH rate observed in the NINDS study. ${ }^{14}$ The Safe Implementation of Thrombolysis in StrokeMonitoring Study (SITS-MOST) has confirmed that intravenous tPA is safe and effective in routine clinical use within $3 \mathrm{~h}$ of stroke onset. ${ }^{15}$ In this observational study of 6483 patients, the proportion of patients with symptomatic ICH was 1.7\% at $24 \mathrm{~h}$ and the mortality rate at 3 months was $11.3 \%$.

\section{PATHOPHYSIOLOGY OF HAEMORRHAGIC TRANSFORMATION}

A haemorrhagic transformation can happen spontaneously, and sometimes from the very first hours at the core of a cerebral infarction. This 
haemorrhagic risk is clearly increased by administration of heparin or thrombolytic agent.

Blood extravasation towards the brain tissue requires an alteration of the blood-brain barrier (BBB), which can occur immediately or be delayed after the ischaemic event. ${ }^{16}$ In experimental models of focal cerebral ischaemia, the basal lamina of the blood vessels and the extracellular matrix are altered and the adhesion between the microvessel cells and the extracellular matrix is compromised. Extravasation of blood elements can then take place. This increase in capillary permeability comes along with an inrush of plasma components inside the brain tissue, an inflammatory reaction with thrombin activation, and implementation of many mediators such as platelet activating factor, tumour necrosis factor $\alpha$ and bradykinin, which contribute to increase endothelial permeability.

Additional vascular lesions related to oxidative damage may increase the haemorrhagic risk. ${ }^{17}$ On this matter, free radical inhibitor pharmacological agents seem efficient towards the reduction in haemorrhagic transformation in experimental conditions. ${ }^{18} 19$

The haemorrhagic transformation also involves the metalloproteinases (MMP) whose activation is partly responsible for the $\mathrm{BBB}$ disruption. In some animal models of focal cerebral ischaemia, activation of MMP-9 is associated with increased permeability of the $\mathrm{BBB}$, with the formation of oedema and haemorrhagic transformation. ${ }^{20} 21$ Some studies have suggested the complementary role of plasmin, which is another serine protease distinct from the MMPs and is generated by interaction between tPA and the thrombus, in the disruption of the $\mathrm{BBB}$ and the occurrence of $\mathrm{ICH} \cdot{ }^{22}$ MMPs are involved in the
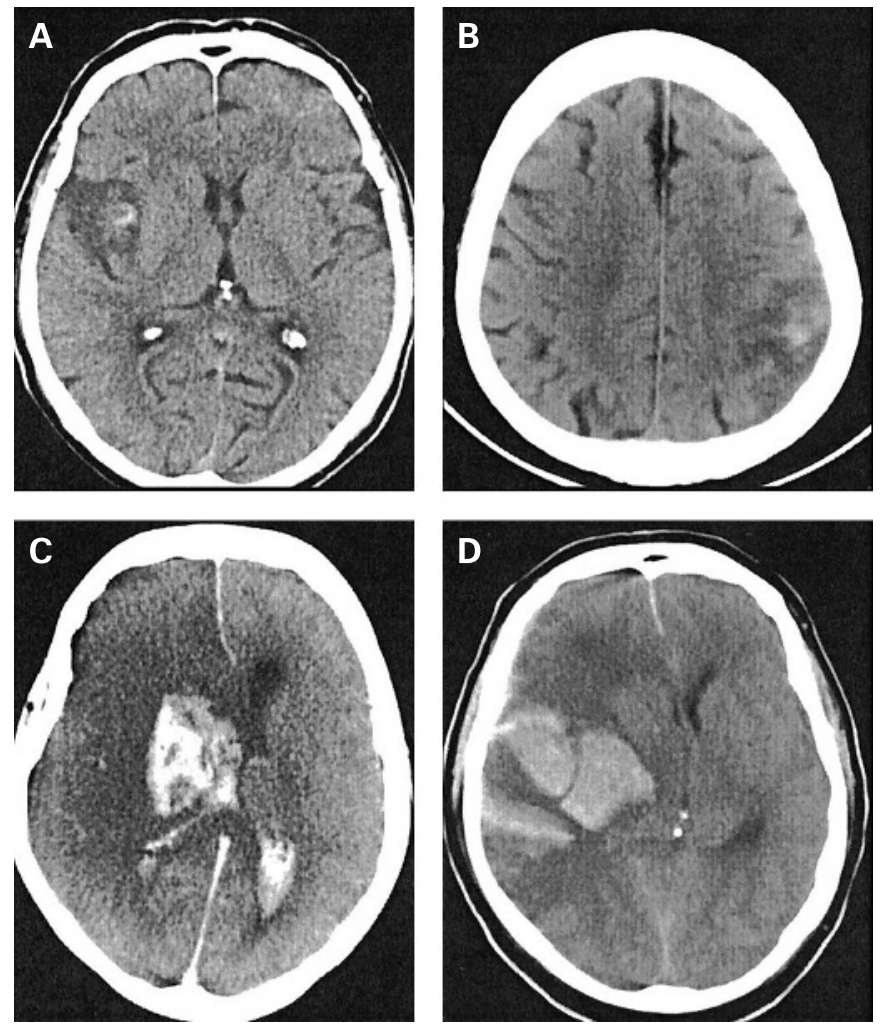

Figure 1 European Cooperative Acute Stroke Study (ECASS) classification of intracerebral haemorrhage (ICH) following thrombolysis (from Berger and colleagues ${ }^{38}$ ). (A) HI-1; (B) HI-2; (C) PH-1; (D) PH-2 (see text for details). genesis of tPA related haemorrhagic transformation. ${ }^{23}$ tPA increased MMP blood levels of the extracellular matrix (MMP9) in a murine model of embolic cerebral ischaemia. MMPs represent a family of proteolytic enzymes combined with zinc, which act normally on the remodelling of the extracellular matrix. Their inappropriate activation can induce proteolysis of the matrix within the neurovascular unity (including the endothelium, astrocyte and neuron). MMPs liberated by the endothelium and the polynuclears at the inflammatory stage of ischaemia use type IV collagen and laminin as substrates. ${ }^{24}$ MMP-2 and MMP-9 can damage the proteic vessel components, especially type IV collagen, fibronectin and laminin, comprising the basal lamina of the cerebral vessels. In humans, elevation of MMP-9 is linked to the severity of the ischaemic stroke, ${ }^{25}$ and the pre-therapeutic MMP-9 rate is an independent predictor of the risk of haemorrhagic transformation related to thrombolysis. ${ }^{26}{ }^{27}$ The association of non-specific inhibitor of MMPs (BB94) with tPA could limit the frequency of haemorrhagic transformation, without diminishing lytic efficiency. ${ }^{24} 28$

Other elements take part in the genesis of tPA related $\mathrm{ICH}$ : factors predisposing to the microangiopathic process and to leukoaraiosis, such as age, hypertension, diabetes mellitus or cerebral amyloid angiopathy, as well as the intensity of the ischaemic process itself, which is evident by the extent of early ischaemic signs shown on brain CT $\operatorname{scan}^{629-31}$ or the volume of cerebral ischaemic lesions on diffusion weighted MRI sequence. $^{11}$

\section{CLASSIFICATION AND IMAGING METHODS OF INTRACEREBRAL HAEMORRHAGES AFTER THROMBOLYSIS}

The two most commonly used classifications are the NINDS and ECASS; both are founded on brain CT scan data.

\section{NINDS classification}

Haemorrhagic transformations are classified into two radiological groups: haemorrhagic cerebral infarctions and intracerebral haematomas. ${ }^{4}$ Haemorrhagic cerebral infarction is defined as CT findings of acute infarction with punctate or variable hypodensity/hyperdensity with an indistinct border within the vascular territory suggested by acute neurological signs and symptoms. Intracerebral haematoma is defined as CT findings of a typical homogeneous, hyperdense lesion with a sharp border with or without oedema or mass effect within the brain. This hyperdense lesion could arise at a site remote from the vascular territory of the ischaemic stroke or within but not necessarily limited to the territory of the presenting cerebral infarction. Haemorrhage with an intraventricular extension is considered an intracerebral haematoma.

\section{ECASS classification}

This classification had initially been used in the ECASS I study ${ }^{32}$ and then reapplied in the ECASS II study. ${ }^{6}$ It is a purely radiological definition subdivided into four categories (fig 1): HI1 , haemorrhagic infarction type 1 (small petechiae along the margins of the infarct); HI-2, haemorrhagic infarction type 2 (confluent petechiae within the infracted area, but without space-occupying effect); $\mathrm{PH}-1$, parenchymal haematoma type 1 (a haematoma in $\leqslant 30 \%$ of infarcted area with some slight space occupying effect); $\mathrm{PH}-2$, parenchymal haematoma type 2 (a dense haematoma $>30 \%$ of the infarcted area with substantial space occupying effect, or as any haemorrhagic lesion outside the infarcted area). 


\section{Imaging methods of intracerebral haemorrhage}

CT scan remains the most widely used imaging method to exclude the presence of intracerebral haemorrhagic lesions. However, it has been shown that MRI may be as accurate as CT for the detection of acute haemorrhage in patients presenting with acute focal stroke symptoms and is more accurate than CT for the detection of chronic intracerebral haemorrhage. ${ }^{33} \mathrm{~T}^{*}$ gradient echo MRI sequences are sensitive to the magnetic susceptibility artefacts generated by the haemoglobin degradation products (deoxyhaemoglobin, methaemoglobin and haemosiderin). These paramagnetic substances modify the magnetic relaxation properties on $\mathrm{T} 2$ and lead to dephasing of the signal on $\mathrm{T} 2 *{ }^{34}$

An advantage of MRI over CT is the ability of T2* sequences to detect small ( $<5 \mathrm{~mm}$ in diameter) focal homogeneous areas of signal loss corresponding to "microbleeds". These "microbleeds" correspond to old asymptomatic microhaemorrhages; a histopathological and MRI correlation analysis of 11 cerebral specimens showed that these hyposignals on MRI were linked to the presence of haemosiderin perivascular deposits. ${ }^{35}$

\section{SYMPTOMATIC OR ASYMPTOMATIC HAEMORRHAGE?}

In the NINDS study as well as in some meta-analyses, symptomatic ICH was defined as a CT documented haemorrhage that was temporally related to any deterioration in the patient's clinical condition in the judgment of the clinical investigator. ${ }^{4}$

However, the most common definition of symptomatic haemorrhage in stroke thrombolytic trials is haemorrhage associated with worsening by 4 or more points on the NIHSS score. $^{936}$

This definition is questionable because it does not take into account the importance of the ICH observed on CT. The clinical deterioration could be a consequence of aggravation of the cerebral infarction itself coinciding with a haemorrhagic transformation, which may only concern a small part of the ischaemic lesion. This definition also neglects other factors, such as the importance of the ischaemic oedema or the associated mass effect that could be more responsible for the neurological deterioration than the haemorrhage itself.

Of course not all post-thrombolysis ICHs have the same clinical significance. The post-thrombolysis ICH spectrum ranges from frequent benign haemorrhagic petechiae, some of which are associated with early reperfusion, ${ }^{37}$ to rare large parenchymal haematomas with severe mass effect. A post hoc analysis from the ECASS II study showed that only the PH-2 type parenchymal haematomas (homogeneous haematomas with mass effect occupying $>30 \%$ of the ischaemic lesion volume) were independently associated with clinical deterioration and poor prognosis. ${ }^{38}$ The ECASS I study had already shown that the risks of early neurological deterioration and death within 3 months were severely increased in cases where $\mathrm{PH}-2$-type haematomas appeared (odds ratio (OR) 32.3 (95\% confidence interval (CI) 13.4 to 77.7$)$ and 18 (95\% CI 8.05 to 40.1), respectively), this type of haematoma being the only one likely to aggravate the clinical status. ${ }^{32}$

\section{CLINICAL, BIOLOGICAL, AND IMAGING PREDICTORS OF ICH \\ Timing of thrombolysis}

Longer stroke onset to treatment times may be associated with higher rates of symptomatic ICH. ${ }^{39}$ However, the vast majority of studies have not identified time to onset of thrombolytic treatment as an independent risk factor of symptomatic ICH in multivariate analysis, and the pooled analysis of the NINDS, ECASS and ATLANTIS trials showed no independent association between timing of tPA administration and risk of parenchymal haematoma. ${ }^{40}$

\section{Lytic dose}

The early pilot trials of tPA have provided good evidence that higher doses of lytic agents lead to higher rates of symptomatic ICH. ${ }^{41}$ The high rates of symptomatic bleeding observed in the streptokinase acute stroke trials may be caused in part by the use of the full cardiac dose of streptokinase. ${ }^{8}$ Dose escalation studies of the newer generation fibrinolytic agents, desmoteplase and tenecteplase, also show increased rates of cerebral bleeding with higher lytic doses. ${ }^{42} 43$

\section{Age}

Several studies ${ }^{41} 30{ }^{31}$ have shown that age is a predictive factor of tPA related ICH. In the ECASS I study, it is the only independent predictive factor of parenchymal haematomas, with an OR of 1.3 for every 10 year increment in age increase. ${ }^{30}$ This increase in the haemorrhagic risk could be linked to a particularly high frequency of microangiopathic processes, especially the cerebral amyloid angiopathy in elderly patients. ${ }^{44}$ This angiopathy is a well known risk factor for tPA related parenchymal haematomas. ${ }^{45}$

In some European countries, tPA is not recommended for the treatment of cerebral infarction in subjects over the age of 80 years, which can lead to exclusion of up to $30 \%$ of ischaemic stroke patients. The prospective multicentre Canadian Alteplase for Stroke Effectiveness Study (CASES), recently completed in Canada, shows that the benefit-risk ratio of intravenous tPA can be favourable in carefully selected elderly stroke patients who are treated within $3 \mathrm{~h} .{ }^{46}$ The symptomatic ICH rate was $4.4 \%$ in the group of 270 patients aged 80 years or older included in this study. The Stroke Survey Group tPA analysis, which included a small number of aged patients treated by intravenous tPA within $3 \mathrm{~h}$, also concluded that it was not justified to systematically contraindicate thrombolysis for patients aged 80 years or older. ${ }^{47}$ Data from randomised studies performed in elderly patients are not currently available and these favourable results from open studies could be altered by selection bias.

\section{Clinical stroke severity}

Many studies have shown the importance of the initial clinical stroke severity in the haemorrhagic risk after thrombolysis. $^{48113148} 49$ In the NINDS study, patients with an NIHSS score of $>20$ at admission were 11 times more likely to endure a symptomatic ICH than patients with an NIHSS score $\leqslant 5 .{ }^{4}$ The Multicentre tPA Acute Stroke Survey study also showed that the NIHSS score was an independent marker of ICH, with an OR of 1.38 for a 1 point raise in the NIHSS score. ${ }^{49}$ In some countries, a NIHSS score $>25$ represents a contraindication for intravenous tPA.

\section{Blood pressure}

In our experience, hypertension during the first $24 \mathrm{~h}$ after ischaemic stroke is an independent predictive factor of any ICH following tPA administration. ${ }^{11}$ This factor has already been emphasised in other thrombolysis studies conducted for cerebral infarction ${ }^{4149}$ or myocardial infarction. ${ }^{50}$ These data underline the importance of a close watch of blood pressure $(\leqslant 185 /$ $110 \mathrm{~mm} \mathrm{Hg}$ ) during the first $24 \mathrm{~h}$ and the benefit of applying a 
strict therapeutic protocol for the control of elevated blood pressure, similar to the one used by the NINDS study, ${ }^{1}$ in order to limit the haemorrhagic risk.

\section{Hyperglycaemia}

Several studies have shown the influence of pre-therapeutic glycaemia on the risk of ICH after stroke thrombolysis. $^{10} 11495152$ In a study performed in 138 consecutive patients with ischaemic stroke, those with a glycaemia value of $>200 \mathrm{mg} / \mathrm{dl}(11.11 \mathrm{mmol} / \mathrm{l})$ on admission showed a symptomatic haematoma rate of $25 \%$ after treatment by tPA. ${ }^{51}$ In the PROACT II study, there was also an increased risk of symptomatic ICH in patients with a pre-therapeutic glycaemia value of $>200 \mathrm{mg} / \mathrm{dl} .^{9}$

There are numerous animal experimental proofs that hyperglycaemia provokes microvascular lesions as well as some $\mathrm{BBB}$ damage, leading to haemorrhagic transformation of the cerebral infarction. ${ }^{53}$ Administration of tPA leads to upregulation of MMPs which aggravate the ischaemic damage of the basal lamina, leading to loss of the integrity of the vessel wall and to $\mathrm{ICH}^{53}$ This deleterious effect of tPA is emphasised in cases of hyperglycaemia. Careful attention must be given to the pre-therapeutic glycaemia value. A glycaemia value above $400 \mathrm{mg} / \mathrm{dl}(22.22 \mathrm{mmol} / \mathrm{l})$ represents a contraindication to cerebral thrombolysis by intravenous tPA.

\section{MMP-9, PAI-1, TAFI and cFN: new biomarkers of the haemorrhagic risk?}

The roles of different biomarkers in the post-thrombolysis ICH risk still need to be clarified. One study showed that increased MMP-9 levels prior to administration of tPA increase the risk of parenchymal haemorrhage. ${ }^{26}$ The importance of plasma levels of MMP-9 has to be clarified to determine if measurement of pretreatment MMP-9 levels is likely to improve the benefit-risk ratio of tPA administration for stroke in routine use. ${ }^{27}$ The relationship between elevation in MMP-9 plasma levels and modifications of $\mathrm{BBB}$ permeability on MRI has not been established in humans at the acute stage of cerebral ischaemia. Demonstration of a causal link between MMP-9 plasma levels and tPA related ICH risk is likely to generate a preventive approach by neutralisation of MMP-9 activity in clinical practice. Administration of an MMP inhibitor decreased the rate of haemorrhagic transformation following tPA administration in a rabbit stroke model..$^{28}$

Some believe that the plasma elevation of cellular fibronectin (cFN), a substance synthesised by endothelial cells that reflects microvascular damage, would be a more specific marker than MMP-9 concentration of tPA related $\mathrm{ICH} .{ }^{54}$

Preliminary studies have also suggested that there are several other biomarkers that may help predict the risk of ICH. Pretreatment levels of endogenous fibrinolysis inhibitors (plasminogen activator inhibitor (PAI-1) and thrombin activated fibrinolysis inhibitor (TAFI)) could predict tPA related symptomatic ICH. ${ }^{55}$ One pilot study demonstrated an independent association between low levels of PAI-1 and tPA related symptomatic $\mathrm{ICH}^{55}$ whereas another recent study found no such association. ${ }^{39}$

\section{Brain CT scan}

\section{Early Ischaemic changes}

The significance of early ischaemic changes (EIC) on baseline brain CT scan remains controversial..$^{56}$ The ECASS I and ECASS II studies have shown that the presence of EIC occupying more than one-third of the middle cerebral artery (MCA) territory before thrombolysis by intravenous tPA is accompanied by an increase in the hemorrhagic transformation risk (especially of $\mathrm{PH}$ 2 type in the ECASS II study) and poor clinical outcome. ${ }^{629-31} 39$ The multicentre analysis of 1205 patients routinely treated by intravenous tPA within $3 \mathrm{~h}$ shows that the symptomatic ICH rate is multiplied by more than 4 in patients carrying EIC occupying more than one-third of the MCA territory. ${ }^{49}$ In the MAST-E study, the presence of EIC was also a strong predictor of haemorrhagic transformation and symptomatic $\mathrm{ICH}^{8}$ In contrast, other thrombolysis studies have shown that the presence of EIC was related to stroke severity but was not independently associated with the occurrence of side effects. ${ }^{58} 59$ The ASK (Australian Streptokinase Trial) study, evaluating treatment with intravenous streptokinase within the first $4 \mathrm{~h}$ of ischaemic stroke, did not show a significant association between the presence of EIC and the occurrence of a major $\mathrm{ICH}^{60}{ }^{60}$

Moreover, the sensitivity and reproducibility of EIC detection are poor and depend on the quality of the CT scanner used and on the experience of the reader. ${ }^{61}$ More specifically, appreciation of the presence of EIC occupying more than one-third of the MCA territory is dependent on the operator and is not reproducible. ${ }^{62}$ Interobserver reproducibility can be improved by a standardised reading of the EIC according to the ASPECTS score (Alberta Stroke Program Early CT Score). ${ }^{63}$

\section{Hyperdense middle cerebral artery sign}

We have shown that the presence of an arterial hyperdensity on the pre-therapeutic brain CT scan is an independent predictive factor of any tPA related ICH. ${ }^{11}$ It was previously demonstrated that this sign was frequently observed in patients developing an asymptomatic ICH after thrombolysis by intravenous tPA within the first 3 h. ${ }^{49}$ However, the low sensitivity (30\%) of the hyperdense MCA sign may limit its usefulness in the prediction of outcome. ${ }^{64}$ In our series, the presence of an arterial hyperdensity on brain CT scan is associated with MCA or internal carotid artery occlusion in most cases and with a specific MRI pattern consisting of a large pretreatment diffusion and perfusion abnormality volume. ${ }^{11}$ The presence of a proximal arterial hyperdensity, as the severity of perfusion reduction suggests, is probably associated with a limited collateral blood supply. The maximal haemodynamic consequence lies in the territory of the perforating lenticulo-striate arteries and the severity of the ischaemic injury in this territory probably helps the haemorrhagic transformation. ${ }^{16}$

\section{Value of MRI data}

Data concerning the input of pre-therapeutic multimodal MRI sequences in the prediction of ICH risk are limited, and usually based only on analysis of diffusion and T2* sequences. ${ }^{11}{ }^{65-69}$ Identification of MRI predictors of ICH could improve the selection of candidates for stroke thrombolysis and lead to a safer use of tPA after $3 \mathrm{~h}$.

\section{Apparent diffusion coefficient}

Recent studies have suggested that diffusion weighted imaging (DWI) sequences could help in identifying patients at risk of ICH. ${ }^{65-67}$ We observed a significant influence of the pretherapeutic values of the apparent diffusion coefficient (ADC) on $\mathrm{ICH}$ risk after thrombolysis by intravenous tPA. ${ }^{11}$ A greater percentage of pixels had a low ADC coefficient $\left(<400 \times 10^{-6} \mathrm{~mm}^{2} / \mathrm{s}\right)$ in patients who had bled compared with patients who had not bled. Tong et al also studied the influence 
of $\mathrm{ADC}$ values on hemorrhagic risk. ${ }^{65}$ In this retrospective study of 17 patients with ischaemic stroke who had DWI sequences within the first $8 \mathrm{~h}, 11$ had been treated with intravenous or intra-arterial tPA. A significantly greater percentage of pixels had low $\mathrm{ADC}$ values in the ischaemic lesions subject to bleeding in comparison with the ischaemic lesions not subject to bleeding. The authors suggested that $\mathrm{a} \leqslant 550 \times 10^{-6} \mathrm{~mm}^{2} / \mathrm{s}$ threshold allowed them to distinguish the ischaemic lesions subject to bleeding. Selim et al also showed that the absolute volume of ischaemic tissue on DWI sequences with $\mathrm{ADC}$ values $\leqslant 550 \times 10^{-6} \mathrm{~mm}^{2} / \mathrm{s}$ was significantly associated with ICH risk after intravenous tPA. ${ }^{67}$

The feasibility of the emergency analysis of ADC values will have to be prospectively evaluated in the future. However, a pre-therapeutic evaluation of the severity of the ischaemic injury based on the precise analysis of the ADC values could be useful if thrombolysis is considered beyond the first $3 \mathrm{~h}$.

\section{Diffusion weighted imaging and perfusion weighted imaging}

Alsop et al sought to define characteristics of haemodynamic MRI that best predict haemorrhage. ${ }^{70}$ Bolus contrast and diffusion MRI were performed before intravenous tPA therapy in 20 patients presenting with acute stroke symptoms within the first $6 \mathrm{~h}$ after symptom onset. Of the 20 patients studied, five had detectable haemorrhage on follow-up scans. Blood volume maps demonstrated virtually no signal within much of the haemorrhagic region, indicating that contrast did not arrive by the end of the imaging series.

Recently, a prospective, multicentre study (Diffusion and Perfusion Imaging Evaluation For Understanding Stroke Evolution (DEFUSE) study) was performed in 74 consecutive stroke patients admitted to academic stroke centres in North America and Europe. ${ }^{71}$ An MRI scan was obtained immediately before and 3-6 h after treatment with intravenous tPA, 3-6 h after symptom onset. Early reperfusion was associated with fatal ICH in patients with the "malignant profile" (baseline DWI volume $\geqslant 100 \mathrm{ml}$ and/or perfusion weighted imaging deficit $\geqslant 100 \mathrm{ml}$ with $8 \mathrm{~s}$ or longer of Tmax delay). Patients with large baseline DWI lesion volumes who achieve early reperfusion appear to be at greatest risk of tPA related symptomatic ICH. ${ }^{72}$ DEFUSE is the first study that directly established a strong relationship between MRI haemodynamic and tissue parameters and symptomatic tPA related ICH.

\section{Blood-brain barrier disruption}

$\mathrm{BBB}$ disruption helping haemorrhagic transformation after thrombolysis is currently being evaluated with MRI. MRI assessments of $\mathrm{BBB}$ permeability include hyperintense acute reperfusion marker, a delayed gadolinium enhancement of the CSF space on fluid attenuated inversion recovery imaging, and permeability imaging. ${ }^{73} 74$

\section{Abnormal visibility of transcerebral veins}

Some preliminary data suggest that abnormal visibility of the transcerebral veins among the ischaemic zone on the pretherapeutic $\mathrm{T}^{*}$ gradient echo MRI sequence could be a predictor of $\mathrm{ICH}$ after thrombolysis of internal carotid artery territory stroke. ${ }^{75}$ This abnormal visibility of the transcerebral veins is related to a more severe neurological deficit, as assessed by the NIHSS score, a proximal site of arterial occlusion, a larger lesion volume on the DWI and perfusion weighted imaging sequences, and a delay in the mean transit time among the perfusion abnormality.
This association between abnormal visibility of the transcerebral veins and post-thrombolysis ICH was later supported by angiographic data. ${ }^{76}$ Ohta et al prospectively studied 104 patients who underwent superselective local angiography before and during intra-arterial reperfusion therapy for acute MCA occlusion. There was a significant correlation between early venous filling and massive haematoma in both the deep and superficial MCA territories. None of the 31 ischaemic areas with early venous filling could escape cerebral infarction. Appearance of early venous filling may indicate irreversible brain damage and may be a predictive sign for parenchymal haematoma.

\section{Leukoaraiosis}

A recent retrospective multicentre study evaluated whether leukoaraiosis is a risk factor for symptomatic ICH in 449 patients treated with thrombolysis for anterior circulation stroke less than $<6 \mathrm{~h}$ after symptom onset. ${ }^{77}$ All patients had received MRI evaluation before thrombolysis, including a high quality T2 weighted sequence. For the analysis, leukoaraiosis in the deep white matter was dichotomised into absent or mild versus moderate or severe. The rate of symptomatic ICH was significantly higher in patients with moderate to severe leukoaraiosis of the deep white matter ( $n=12$ of $114 ; 10.5 \%)$ than in patients without relevant leukoaraiosis ( $n=13$ of $335 ; 3.8 \%$ ), corresponding to an OR of 2.9 (95\% CI 1.29 to 6.59; $p=0.015)$. In a logistic regression analysis (including age, NIHSS score on admission and type of thrombolytic treatment), leukoaraiosis remained an independent risk factor (OR 2.9; $\mathrm{p}=0.03$ ).

\section{Presence of microbleeds}

The risk of ICH after thrombolysis in ischaemic stroke patients carrying old asymptomatic microbleeds remains a controversial subject. ${ }^{78-80}$ The presence of microbleeds represents a marker of microangiopathy and may indicate an increased risk of haemorrhagic transformation after ischaemic stroke. ${ }^{68} 78$ However, data concerning the safety of thrombolysis in patients carrying microbleeds remain limited..$^{61-83}$

Few thrombolysis studies have performed pre-therapeutic T2* MRI sequences to evaluate the influence of the presence of microbleeds on ICH risk. Kidwell et al have reported the case of a patient who had a symptomatic haematoma inside a microbleed after treatment by intra-arterial thrombolysis of a cerebral infarction contralateral to the bleeding. ${ }^{68}$ In our series, eight (18\%) of the 44 acute ischaemic stroke patients included in a thrombolysis protocol showed some microbleeds on the pretherapeutic T2* MRI sequence. ${ }^{81}$ A similar rate of $16 \%$ of patients carrying microbleeds before thrombolysis has recently been reported.$^{82}$ In our study, none of the eight patients carrying microbleeds had a haemorrhage at the site of a microbleed and none showed signs of a symptomatic ICH at day 1 . No cerebral haemorrhage occurred outside the infarction core. ${ }^{81}$ These data underline the role of the ischaemic injury in the genesis of haemorrhagic transformation following tPA administration. In the NINDS study, only four symptomatic ICHs occurred outside the area of the cerebral infarction among patients treated with tPA (ie, $1.3 \%$ of all patients treated by tPA). ${ }^{4}$ Kakuda et al have also treated 11 stroke patients carrying microbleeds by intravenous tPA within 3-6 h after symptom onset. ${ }^{82}$ None of the patients experienced symptomatic or asymptomatic ICH at the site of a microbleed. In a recent pooled analysis of 570 patients, the presence of microbleeds (detected in 86 patients) was not predictive of symptomatic ICH after thrombolysis. ${ }^{83}$ 
The limited data currently available do not allow for exclusion of stroke patients carrying a few microbleeds $(<5)$ on pre-therapeutic $2^{*}$ sequences from thrombolysis with intravenous tPA. ${ }^{68}{ }^{81-83}$ Additional data are needed to evaluate the safety of tPA in patients carrying numerous microbleeds. The data extracted from international collaborative multicentre studies will assist in clarifying this issue.

\section{CONCLUSION}

ICH after stroke thrombolysis is a complex and heterogeneous phenomenon, which involves multiple demographic, clinical, biological and haemodynamic parameters, whose knowledge remains partial. In order to minimise the risk of tPA related symptomatic ICH, careful attention must be given to the pretherapeutic glycaemia value, ${ }^{49} 51$ and a strict protocol for the control of elevated blood pressure is needed during the first $24 \mathrm{~h}$ following thrombolysis. ${ }^{1} 49$

Multicentre studies will bring useful information on the predictors of symptomatic ICH, which is the most important type of haemorrhagic transformation after stroke thrombolysis from a clinician's point of view. Some authors have suggested that the differences between symptomatic and asymptomatic ICHs are more linked to the intensity of the bleeding than to physiopathological differences. ${ }^{16}$ For other authors, haemorrhagic infarctions and parenchymal haematomas (according to the ECASS classification) after tPA have a different clinical, aetiological and biological significance. ${ }^{12}{ }^{84}$ Benign haemorrhagic infarctions could be associated with the natural evolution of cerebral infarction while parenchymal haematomas, especially the PH-2 type, severe by their immediate or delayed symptomatic character, could be linked to the tPA itself and particularly to its impact on haemostasis (elevation of fibrin degradation products (FDP) after treatment). ${ }^{85}$

Adherence to the inclusion criteria of the NINDS study remains the key to a favourable benefit-risk ratio in the routine use of tPA for stroke. However, these inclusion criteria are now more than 10 years old and can appear obsolete as they do not take into account the diagnostic and prognostic input of pretherapeutic MRI. The input of multimodal MRI sequences and of the new biomarkers (MMP-9, PAI-1, TAFI and cFN) of ICH risk in the appreciation of the benefit-risk ratio will have to be clarified in the future. If some MRI patterns ("malignant profile" with baseline DWI volume $\geqslant 100 \mathrm{ml}$ ) associated with an increased risk of severe ICH or with the absence of "salvageable" brain tissue represent real contraindications to stroke thrombolysis, the safety of tPA could be significantly improved. A recent multicentre study compared the clinical evolution of patients selected on the basis of MRI and treated by intravenous tPA within the first $6 \mathrm{~h}$ to that of patients selected on the basis of brain CT scan and included in the ATLANTIS, ECASS and NINDS studies. ${ }^{86}$ The decision to treat between 3 and $6 \mathrm{~h}$ was based on the judgment of the clinician after analysis of the MRI data. Generally, patients without significant perfusion-diffusion mismatch and patients with an extended lesion on the diffusion sequence $(>50 \%$ of the MCA territory) were not treated with thrombolysis. The rate of symptomatic ICH was significantly lower $(3 \%$ vs $8 \%$; $p=0.01)$ and the rate of favourable clinical outcome was significantly higher ( $48 \%$ vs $40 \% ; p=0.05$ ) in the group of patients selected on the basis of MRI. These results are in agreement with those of a recent cohort study evaluating MRI versus CT based thrombolysis treatment within and beyond the $3 \mathrm{~h}$ time window after stroke onset. ${ }^{87}$ MRI selected patients overall had a significantly lower risk than CT selected patients for symptomatic ICH ( $3 \%$ vs $9 \% ; p=0.013)$ and mortality $(12 \%$ vs $21 \% ; p=0.021$. Considering the usual reserves related to the absence of randomisation and the potential bias inherent in this type of study, these data suggest the superiority of an individualised therapeutic decision based on MRI.

Competing interests: None.

\section{REFERENCES}

1. The National Institute of Neurological Disorders and Stroke rt-PA Stroke Study Group. Tissue plasminogen activator for acute ischemic stroke. N Engl J Med 1995; 333:1581-7.

2. Reeves MJ, Arora S, Broderick JP, et al. Acute stroke care in the US: results from the 4 pilot prototypes of the Paul Coverdell National Acute Stroke Registry. Stroke 2005; 36:1232-40.

3. Caplan LR. Stroke thrombolysis: slow progress. Circulation 2006;114:187-90.

4. The National Institute of Neurological Disorders and Stroke rt-PA Stroke Study Group. Intracerebral hemorrhage after intravenous t-PA therapy for ischemic stroke. Stroke 1997:28:2109-18.

5. Clark WM, Wissman S, Albers GW, et al. Recombinant tissue-type plasminogen activator (alteplase) for ischemic stroke 3 to 5 hours after symptom onset: the ATLANTIS Study, a randomized controlled trial: Alteplase Thrombolysis for Acute Noninterventional Therapy in Ischemic Stroke. JAMA 1999;21:2019-26.

6. Hacke W, Kaste M, Fieschi C, et al. Randomised double-blind placebo-controlled trial of thrombolytic therapy with intravenous alteplase in acute ischaemic stroke (ECASS II). Lancet 1998;352:1245-51.

7. Furlan A, Higashida R, Wechsler L, et al. Intra-arterial prourokinase for acute ischemic stroke. The PROACT II Study: a randomized controlled trial. JAMA 1999:282:2003-11.

8. Jaillard A, Cornu C, Durieux A, et al. Hemorrhagic transformation in acute ischemic stroke. The MAST-E Study. Stroke 1999;30:1326-32.

9. Kase CS, Furlan AJ, Wechsler LR, et al. Cerebral hemorrhage after intra-arterial thrombolysis for ischemic stroke. The PROACT II trial. Neurology 2001;57:1603-10.

10. Kidwell CS, Saver JL, Carneado J, et al. Predictors of hemorrhagic transformation in patients receiving intra-arterial thrombolysis. Stroke 2002;33:717-24.

11. Derex L, Hermier $\mathrm{M}$, Adeleine $\mathrm{P}$, et al. Clinical and imaging predictors of intracerebral haemorrhage in stroke patients treated with intravenous tissue plasminogen activator. J Neurol Neurosurg Psychiatry 2005;76:70-5.

12. Trouillas $\mathbf{P}$, von Kummer R. Classification and pathogenesis of cerebral hemorrhages after thrombolysis in ischemic stroke. Stroke 2006:37:556-61.

13. The IMS Study Investigators. Hemorrhage in the Interventional Management of Stroke study. Stroke 2006;37:847-51.

14. Graham GD. Tissue plasminogen activator for acute ischemic stroke in clinical practice: a meta-analysis of safety data. Stroke 2003;34:2847-50.

15. Wahlgren N, Ahmed N, Davalos A, et al. Thrombolysis with alteplase for acute ischaemic stroke in the Safe Implementation of Thrombolysis in Stroke-Monitoring Study (SITS-MOST): an observational Study. Lancet 2007;369:275-82.

16. Del Zoppo GJ, von Kummer R, Hamann GF. Ischaemic damage of brain microvessels: inherent risks for thrombolytic treatment in stroke. J Neurol Neurosurg Psychiatry 1998;65:1-9.

17. Del Zoppo GJ, Zeumer H, Harker LA. Thrombolytic therapy in stroke: possibilities and hazards. Stroke 1986;7:595-607.

18. Hu B, Liu C, Zivin JA. Reduction of intracerebral hemorrhage in a rabbit embolic stroke model. Neurology 1999;53:2140-5

19. Asahi M, Asahi K, Wang X, et al. Reduction of tissue plasminogen activator induced hemorrhage and brain injury by free radical spin trapping after focal cerebral ischemia in rats. J Cereb Blood Flow Metab 2000;20:452-7.

20. Rosenberg GA, Estrada EY, Dencoff JE. Matrix metalloproteinases and TIMPS are associated with blood brain barrier opening after reperfusion in rat brain. Stroke 1998;29:2189-95

21. Asahi M, Wang X, Mori T, et al. Effects of matrix metalloproteinase-9 gene knockout on the proteolysis of blood-brain barrier and white matter components after cerebral ischemia. J Neurosci 2001;21:7724-32.

22. Gautier S, Petrault 0, Gele P, et al. Involvement of thrombolysis in recombinant tissue plasminogen activator-induced cerebral hemorrhages and effect on infarct volume and postischemic endothelial function. Stroke 2003;34:2975-9.

23. Sumii T, Lo EH. Involvement of matrix metalloproteinase in thrombolysis-associated hemorrhagic transformation after embolic focal ischemia in rats. Stroke 2002:33:831-6.

24. Romanic AM, White RF, Arleth AJ, et al. Matrix metalloproteinase expression increases after cerebral focal ischemia in rats. Inhibition of matrix metalloproteinase-9 reduces infarct size. Stroke 1998:29:1020-30.

25. Montaner J, Alvarez-Sabin J, Molina C, et al. Matrix metalloproteinase expression after human cardioembolic stroke: temporal profile and relation to neurologica impairment. Stroke 2001:32:1759-66.

26. Montaner J, Molina CA, Monasterio J, et al. Matrix metalloproteinase-9 pretreatment level predicts intracranial hemorrhagic complications after thrombolysis in human stroke. Circulation 2003;107:598-603.

27. Castellanos M, Leira R, Serena J, et al. Plasma metalloproteinase-9 concentration predicts hemorrhagic transformation in acute ischemic stroke. Stroke 2003:34:40-6. 
28. Lapchak PA, Chapman DF, Zivin JA. Metalloproteinase inhibition reduces thrombolytic (tissue plasminogen activator)-induced hemorrhage after thromboembolic stroke. Stroke 2000;31:3034-40.

29. Hacke W, Kaste M, Fieschi C, et al. Intravenous thrombolysis with recombinant tissue plasminogen activator for acute hemispheric stroke. JAMA 1995;274:1017-26.

30. Larrue V, von Kummer R, del Zoppo G, et al. Hemorrhagic transformation in acute ischemic stroke. Potential contributing factors in the European Cooperative Acute Stroke Study. Stroke 1997;28:957-60.

31. Larrue V, von Kummer R, Müller A, et al. Risk factors for severe hemorrhagic transformation in ischemic stroke patients treated with recombinant tissue plasminogen activator. A secondary analysis of the European-Australasian Acute Stroke Study (ECASS II). Stroke 2001;32:438-41.

32. Fiorelli $\mathbf{M}$, Bastianello $\mathbf{S}$, von Kummer $\mathrm{R}$, et al. Hemorrhagic transformation within 36 hours of a cerebral infarct: relationships with early clinical deterioration and 3month outcome in the European Cooperative Acute Stroke Study I (ECASS I) cohort. Stroke 1999;30:2280-4.

33. Kidwell CS, Chalela JA, Saver JL, et al. Comparison of MRI and CT for detection of acute intracerebral hemorrhage. JAMA 2004;292:1823-30.

34. Unger EC, Cohen MS, Brown TR. Gradient-echo imaging of hemorrhage at 1.5 Tesla. Magn Reson Imaging 1989;7:163-72

35. Fazekas F, Kleinert R, Roob G, et al. Histopathologic analysis of foci of signal loss in gradient-echo $\mathrm{T}^{*}$-weighted MR images in patients with spontaneous intracerebral hemorrhage: evidence of microangiopathy-related microbleeds. AJNR Am J Neuroradiol. 1999;20:637-42.

36. Brott TG, Adams HP Jr, Olinger CP, et al. Measurements of acute cerebral infarction. A clinical examination scale. Stroke 1989:20:864-70.

37. Molina CA, Montaner J, Abilleira S, et al. Time course of tissue plasminogen activator-induced recanalization in acute cardioembolic stroke: a case-control study. Stroke 2001;32:2821-7.

38. Berger C, Fiorelli M, Steiner T, et al. Hemorrhagic transformation of ischemic brain tissue. Asymptomatic or symptomatic? Stroke 2001;32:1330-5.

39. Cocho D, Borrell M, Marti-Fabregas J, et al. Pretreatment hemostatic markers of symptomatic intracerebral hemorrhage in patients treated with tissue plasminogen activator. Stroke 2006:37:996-9.

40. Hacke W, Donnan G, Fieschi C, et al. Association of outcome with early stroke treatment: pooled analysis of ATLANTIS, ECASS, and NINDS rt-PA stroke trials. Lancet 2004:363:768-74.

41. Levy D, Brott T, Haley EC Jr, et al. Factors related to intracranial hematoma formation in patients receiving tissue-type plasminogen activator for acute ischemic stroke. Stroke 1994:25:291-7.

42. Haley EC Jr, Lyden PD, Johnston KC, et al. A pilot dose-escalation safety study of tenecteplase in acute ischemic stroke. Stroke 2005;36:1109-10.

43. Hacke W, Albers G, Al-Rawi Y, et al. The Desmoteplase in Acute Ischemic Stroke Trial (DIAS): a phase II MRI-based 9-hour window acute stroke thrombolysis trial with intravenous desmoteplase. Stroke 2005:36:66-73.

44. Itoh Y, Yamada M, Hayakawa $\mathrm{M}$, et al. Cerebral amyloid angiopathy: a significant cause of cerebellar as well as lobar hemorrhage in the elderly. J Neurol Sci 1993:116:135-41.

45. Vonsattel JP, Myers RH, Hedley-Whyte ET, et al. Cerebral amyloid angiopathy without and with cerebral hemorrhages: a comparative histological study. Ann Neurol 1991;30:637-49.

46. Sylaja PN, Cote R, Buchan AM, et al. Thrombolysis in patients older than 80 years with acute ischaemic stroke: Canadian Alteplase for Stroke Effectiveness Study. J Neurol Neurosurg Psychiatry 2006;77:826-9.

47. Tanne D, Gorman MJ, Bates VE, et al. Intravenous tissue plasminogen activator for acute ischemic stroke in patients aged 80 years and older: the tPA Stroke Survey experience. Stroke 2000;31:370-5.

48. The MAST-Italy Investigators. Risk factors in the MAST-Italy trial. Cerebrovasc Dis 1996;6:181.

49. Tanne D, Kasner SE, Demchuk AM, et al. Markers of increased risk of intracerebral hemorrhage after intravenous recombinant tissue plasminogen activator therapy for acute ischemic stroke in clinical practice. Circulation 2002;105:1679-85.

50. Gore JM, Granger CB, Simoons ML, et al. Stroke after thrombolysis: mortality and functional outcomes in the GUSTO-I Trial. Circulation 1995:92:2811-18.

51. Demchuk AM, Morgenstern LB, Krieger DW, et al. Serum glucose level and diabetes predict tissue plasminogen activator-related intracerebral hemorrhage in acute ischemic stroke. Stroke 1999;30:34-9.

52. Lindsberg PJ, Soinne L, Roine RO, et al. Community-based thrombolytic therapy of acute ischemic stroke in Helsinki. Stroke 2003;34:1443-9.

53. Hamann GF, del Zoppo GJ, von Kummer R. Hemorrhagic transformation of cerebral infarction: possible mechanisms. Thromb Haemost 1999;82:92-4

54. Castellanos M, Leira R, Serena J, et al. Plasma cellular-fibronectin concentration predicts hemorrhagic transformation after thrombolytic therapy in acute ischemic stroke. Stroke 2004;35:1671-6.

55. Ribo M, Montaner J, Molina CA, et al. Admission fibrinolytic profile is associated with symptomatic hemorrhagic transformation in stroke patients treated with tissue plasminogen activator. Stroke 2004;35:2123-7.

56. Von Kummer R. Early major ischemic changes on computed tomography should preclude use of tissue plasminogen activator. Stroke 2003;34:820-1.

57. Lyden P. Early major ischemic changes on computed tomography should not preclude use of tissue plasminogen activator. Stroke 2003;34:821-2.

58. Patel SC, Levine SR, Tilley BC, et al. Lack of clinical significance of early ischemic changes on computed tomography in acute stroke. JAMA 2001:286:2830-8.
59. Roberts HC, Dillon WP, Furlan AJ, et al. Computed tomographic findings in patients undergoing intra-arterial thrombolysis for acute ischemic stroke due to middle cerebral artery occlusion: results from the PROACT II trial. Stroke 2002;33:1565-7.

60. Gilligan AK, Markus R, Read S, et al. Baseline blood pressure but not early computed tomography changes predicts major hemorrhage after streptokinase in acute ischemic stroke. Stroke 2002;33:2236-42.

61. Grotta JC, Chiu D, Lu M, et al. Agreement and variability in the interpretation of early CT changes in stroke patients qualifying for intravenous rtPA therapy. Stroke 1999:30:1528-33.

62. Kalafut MA, Schriger DL, Saver JL, et al. Detection of early CT signs of $>1 / 3$ middle cerebral artery infarctions: interrater reliability and sensitivity of CT interpretation by physicians involved in acute stroke care. Stroke 2000;31:1667-71.

63. Barber PA, Demchuk AM, Zhang J, et al. Validity and reliability of a quantitative computed tomography score in predicting outcome of hyperacute stroke before thrombolytic therapy. ASPECTS Study Group. Alberta Stroke Programme Early CT Score. Lancet 2000;355:1670-4.

64. Leys D, Pruvo JP, Godefroy 0, et al. Prevalence and significance of hyperdense middle cerebral artery in acute stroke. Stroke 1992;23:317-24.

65. Tong DC, Adami A, Moseley ME, et al. Relationship between apparent diffusion coefficient and subsequent hemorrhagic transformation following acute ischemic stroke. Stroke 2000;31:2378-84.

66. Tong DC, Adami A, Moseley ME, et al. Prediction of hemorrhagic transformation following stroke. Arch Neurol 2001;58:587-93

67. Selim M, Fink JN, Kumar S, et al. Predictors of hemorrhagic transformation after intravenous recombinant tissue plasminogen activator: prognostic value of the initial apparent diffusion coefficient and diffusion-weighted lesion volume. Stroke 2002:33:2047-52.

68. Kidwell CS, Saver JL, Villablanca P, et al. Magnetic resonance imaging detection of microbleeds before thrombolysis. An emerging application. Stroke 2002:33:95-8.

69. Oppenheim C, Samson Y, Dormont D, et al. DWI prediction of symptomatic hemorrhagic transformation in acute MCA infarct. J Neuroradiol 2002;29:6-13.

70. Alsop DC, Makovetskaya E, Kumar S, et al. Markedly reduced apparent blood volume on bolus contrast magnetic resonance imaging as a predictor of hemorrhage after thrombolytic therapy for acute ischemic stroke. Stroke 2005:36:746-50.

71. Albers GW, Thijs VN, Wechsler L, et al. Magnetic resonance imaging profiles predict clinical response to early reperfusion: the diffusion and perfusion imaging evaluation for understanding stroke evolution (DEFUSE) study. Ann Neurol 2006;60:508-17.

72. Lansberg MG, Thijs VN, Bammer R, et al. Risk factors of symptomatic intracerebra hemorrhage after tPA therapy for acute stroke. Stroke 2007:38:2275-8.

73. Kassner A, Roberts T, Taylor K, et al. Prediction of hemorrhage in acute ischemic stroke using permeability MR imaging. AJNR Am J Neuroradiol 2005;26:2213-17.

74. Latour LL, Kang DW, Ezzedine M, et al. Early blood-brain barrier disruption in human focal brain ischemia. Ann Neurol 2004;56:468-77.

75. Hermier M, Nighoghossian N, Derex L, et al. Hypointense transcerebral veins at $\mathrm{T2}^{\star}$ weighted MRI: a marker of hemorrhagic transformation risk in patients treated with intravenous tissue plasminogen activator. J Cereb Blood Flow Metab 2003;23:1362-70.

76. Ohta H, Nakano S, Yokogami K, et al. Appearance of early venous filling during intraarterial reperfusion therapy for acute middle cerebral artery occlusion. A predictive sign for hemorrhagic complications. Stroke 2004;35:893-8.

77. Neumann-Haefelin T, Hoelig S, Berkefeld J, et al. Leukoaraiosis is a risk factor for symptomatic intracerebral hemorrhage after thrombolysis for acute stroke. Stroke 2006;37:2463-6.

78. Nighoghossian $\mathbf{N}$, Hermier $\mathrm{M}$, Adeleine $\mathrm{P}$, et al. Old microbleeds are a potential risk factor for cerebral bleeding after ischemic stroke. A gradient-echo T2*-weighted brain MRI study. Stroke 2002;33:735-42.

79. Fan YH, Zhang L, Lam WWM, et al. Cerebral microbleeds as a risk factor for subsequent intracerebral hemorrhages among patients with acute ischemic stroke. Stroke 2003;34:2459-62

80. Chalela JA, Kang DW, Warach S. Multiple cerebral microbleeds: MRI marker of a diffuse hemorrhage-prone state. J Neuroimaging 2004;14:54-7.

81. Derex L, Nighoghossian N, Hermier M, et al. Thrombolysis for ischemic stroke in patients with old microbleeds on pretreatment MRI. Cerebrovasc Dis 2004;17:238-41.

82. Kakuda W, Thijs VN, Lansberg MG, et al. Clinical importance of microbleeds in patients receiving IV thrombolysis. Neurology 2005;65:1175-8.

83. Fiehler J, Albers GW, Boulanger JM, et al. Bleeding risk analysis in stroke imaging before thromboLysis (BRASIL): pooled analysis of T2*-weighted magnetic resonance imaging data from 570 patients. Stroke 2007;38:2738-44.

84. Thomalla G, Sobesky J, Köhrmann M, et al. Two tales: hemorrhagic transformation but not parenchymal hemorrhage after thrombolysis is related to severity and duration of ischemia. MRI study of acute stroke patients treated with intravenous tissue plasminogen activator within 6 hours. Stroke 2007;38:313-18.

85. Trouillas $\mathbf{P}$, Derex L, Philippeau F, et al. Early fibrinogen degradation coagulopathy is predictive of parenchymal hematomas in cerebral rtPA thrombolysis. A study in 157 cases. Stroke 2004:35:1323-8.

86. Thomalla G, Schwark C, Sobesky J, et al. Outcome and symptomatic bleeding complications of intravenous thrombolysis within 6 hours in MRI-selected stroke patients. Comparison of a German multicenter study with the pooled data of ATLANTIS, ECASS, and NINDS tPA trials. Stroke 2006;37:852-8.

87. Köhrmann $\mathbf{M}$, Juttler $\mathrm{E}$, Fiebach JB, et al. MRI versus CT-based thrombolysis treatment within and beyond the $3 \mathrm{~h}$ time window after stroke onset: a cohort study. Lancet Neurol 2006;5:661-7. 